\title{
Precursor Oxidation by Mia40 and Erv1 Promotes Vectorial Transport of Proteins into the Mitochondrial Intermembrane Space
}

\author{
Judith M. Müller, ${ }^{*}$ Dusanka Milenkovic, ${ }^{*}$ Bernard Guiard, ${ }^{+}$Nikolaus Pfanner, ${ }^{*}$ \\ and Agnieszka Chacinska*
}

\begin{abstract}
*Institut für Biochemie und Molekularbiologie, Zentrum für Biochemie und Molekulare Zellforschung, Universität Freiburg, D-79104 Freiburg, Germany; and ${ }^{+}$Centre de Génétique Moléculaire, Centre National de la Recherche Scientifique, F-91190 Gif-sur-Yvette, France
\end{abstract}

Submitted August 22, 2007; Revised October 15, 2007; Accepted October 19, 2007

Monitoring Editor: Donald Newmeyer

\begin{abstract}
The mitochondrial intermembrane space contains chaperone complexes that guide hydrophobic precursor proteins through this aqueous compartment. The chaperones consist of hetero-oligomeric complexes of small Tim proteins with conserved cysteine residues. The precursors of small Tim proteins are synthesized in the cytosol. Import of the precursors requires the essential intermembrane space proteins Mia40 and Erv1 that were proposed to form a relay for disulfide formation in the precursor proteins. However, experimental evidence for a role of Mia40 and Erv1 in the oxidation of intermembrane space precursors has been lacking. We have established a system to directly monitor the oxidation of precursors during import into mitochondria and dissected distinct steps of the import process. Reduced precursors bind to Mia40 during translocation into mitochondria. Both Mia40 and Erv1 are required for formation of oxidized monomers of the precursors that subsequently assemble into oligomeric complexes. Whereas the reduced precursors can diffuse back into the cytosol, the oxidized precursors are retained in the intermembrane space. Thus, oxidation driven by Mia40 and Erv1 determines vectorial transport of the precursors into the mitochondrial intermembrane space.
\end{abstract}

\section{INTRODUCTION}

About 1000 different precursor proteins are imported into mitochondria, and they are sorted to the four mitochondrial subcompartments: outer and inner membranes, intermembrane space (IMS), and matrix (Sickmann et al., 2003; Prokisch et al., 2004). To fulfill this task, four main pathways of protein translocation into mitochondria exist (Jensen and Johnson, 2001; Matouschek and Glick, 2001; Hoogenraad et al., 2002; Endo et al., 2003; Dolezal et al., 2006; Bohnert et al., 2007; Milenkovic et al., 2007b; Neupert and Herrmann, 2007). The precursor proteins enter mitochondria via the translocase of the outer mitochondrial membrane (TOM) and subsequently follow specific pathways leading to the various destinations. 1) Amino-terminal presequences direct precursor proteins across the inner mitochondrial membrane into the matrix via the presequence translocase of the inner membrane (TIM23 complex). 2) Multispanning hydrophobic proteins of the inner membrane that are synthesized without presequences are guided across the aqueous environment of

This article was published online ahead of print in $M B C$ in Press (http:/ / www.molbiolcell.org/cgi/doi/10.1091/mbc.E07-08-0814) on October 31, 2007

Address correspondence to: Nikolaus Pfanner (nikolaus.pfanner@ biochemie.uni-freiburg.de).

Abbreviations used: AMS, 4-aceto-4' ${ }^{\prime}$-maleimidylstilbene-2,2'-disulfonic acid; DTT, dithiothreitol; IA, iodoacetamide; IMS, intermembrane space; MIA, mitochondrial intermembrane space import and assembly; SAM, sorting and assembly machinery; TIM, translocase of inner membrane; TOM, translocase of outer membrane. the IMS by the TIM chaperone complexes Tim9-Tim10 and Tim8-Tim13. Subsequently, the precursor proteins are recognized by the inner membrane carrier translocase (TIM22 complex) that drives their insertion into the inner membrane. 3) $\beta$-Barrel proteins of the outer membrane are transferred from the TOM complex to the sorting and assembly machinery (SAM complex) of the outer membrane with the help of the TIM chaperone complexes. SAM mediates the membrane insertion of the $\beta$-barrel precursors. 4) Precursors of small IMS proteins pass through the TOM complex, and they are directed into the IMS by the mitochondrial IMS import and assembly machinery (MIA).

Although numerous components of the mitochondrial protein import machinery are known, the mechanisms operating along the import pathways are only partially understood. The pathways for proteins with a presequence and inner membrane carrier protein rely on two main driving forces to reach the inner mitochondrial membrane, that is, a chain of increasing binding affinities to the consecutive import components and the electrochemical gradient. To complete translocation across the inner membrane into the mitochondrial matrix, the presequence-carrying precursors use ATP as an additional energy source to drive mitochondrial 70-kDa heat-shock protein, a major component of the presequence translocase-associated motor (PAM complex) (Jensen and Johnson, 2001; D'Silva et al., 2003; Rehling et al., 2004; Chacinska et al., 2005; Oka and Mihara, 2005; Neupert and Herrmann, 2007). In contrast, very little is known about the driving forces operating along the more recently discovered $\beta$-barrel (SAM) and MIA pathways for the precursors targeted to the outer membrane and the IMS, respectively. 
The majority of IMS proteins are small proteins with conserved cysteine motifs. Representatives of the IMS proteins are soluble members of the family of small Tim proteins, Tim8, Tim9, Tim10, and Tim13, which contain a conserved twin Cys- $X_{3}$-Cys motif (Koehler et al., 1998, 1999; Sirrenberg et al., 1998; Adam et al., 1999; Koehler, 2004). The small Tim proteins are assembled into hexameric complexes, Tim9-Tim10 and Tim8-Tim13 (Adam et al., 1999; Curran et al., 2002a,b; Vial et al., 2002; Lu et al., 2004b; Webb et al., 2006). These complexes function as chaperones that guide hydrophobic precursors of $\beta$-barrel proteins and carrier proteins through the IMS (Paschen et al., 2000; Davis et al., 2000, 2007; Luciano et al., 2001; Curran et al., 2002a,b; Hoppins and Nargang, 2004; Koehler, 2004; Wiedemann et al., 2004, 2006). The cysteine residues of small Tim proteins are essential for import and assembly of the mature complexes. It has been debated whether the crucial role of the cysteine residues was due to formation of disulfide bonds or binding of metal ions. Binding of zinc ions likely maintains the cytosolic precursors of small Tim proteins in a reduced and import-competent state (Lu et al., 2004a; Lu and Woodburn, 2005). In addition, however, it was reported that the cysteine residues of the mature TIM chaperone complexes coordinate zinc ions (Sirrenberg et al., 1998; Lutz et al., 2003). This finding has been challenged by a number of observations in vitro and in organello that the cysteine residues of the folded subunits and the mature TIM complexes exist in an oxidized state, i.e., form intramolecular disulfide bonds (Curran et al., 2002a,b; Allen et al., 2003; Lu et al., 2004a). The presence of intramolecular disulfide bonds in each subunit was indeed directly demonstrated by the high-resolution structure of the Tim9-Tim10 complex (Webb et al., 2006).

The biogenesis of IMS proteins requires the essential proteins Mia40 and Erv1 that comprise the MIA-system. The first critical step of sorting into the IMS is a direct association of precursor proteins with Mia40 upon their transfer through the TOM complex (Chacinska et al., 2004; Naoé et al., 2004; Terziyska et al., 2005, 2007; Gabriel et al., 2007; Milenkovic et al., 2007a; Sideris and Tokatlidis, 2007). Mia40 determines the specificity of substrate entry into the IMS by selective binding to specific cysteine residues of the precursors, thus performing a receptor-like function (Milenkovic et al., 2007a; Sideris and Tokatlidis, 2007). Erv1 is a sulfhydryl oxidase (Lee et al., 2000). It interacts with Mia40 and keeps it in an oxidized state (Allen et al., 2005; Mesecke et al., 2005; Rissler et al., 2005). Different views exist if Erv1 is also required for the release of precursors from Mia40 (Mesecke et al., 2005; Rissler et al., 2005). A hypothesis has been formulated that Erv1, Mia40, and IMS precursors form a disulfide relay. Such a relay might resemble the reaction cascades that exist in the endoplasmic reticulum and bacterial periplasm, involving Ero1/Erv2-Pdi1 and DsbB-DsbA, respectively (Collet and Bardwell, 2002; Kadokura et al., 2003; Tu and Weissman, 2004; Wilkinson and Gilbert, 2004; Sevier and Kaiser, 2006). The reactions result in the transfer of disulfides into the unfolded precursor proteins. The identification of transient intermolecular disulfide bonds (mixed disulfides) between Erv1 and Mia40 and between Mia40 and precursor proteins supports the model of a regulated transfer of disulfide bonds (Chacinska et al., 2004; Mesecke et al., 2005; Rissler et al., 2005; Milenkovic et al., 2007a,b; Sideris and Tokatlidis, 2007). However, experimental evidence is lacking that the MIA-system indeed oxidizes the precursor proteins, i.e., transfers disulfide bonds into the precursors. Moreover, although the disulfide relays in the endoplasmic reticulum and bacteria are not coupled to protein translocation across membranes, the MIA-system is required for the translocation of proteins into the IMS, but the molecular mechanism determining vectorial transport has not been elucidated.

Here, we have established an in organello assay to monitor the oxidation process of the precursors of Tim8 and Tim13 on their assembly pathway into the IMS. We identified a pool of oxidized precursor monomers that function as intermediates in formation of the Tim8-Tim13 chaperone complex and defined four functional import stages: reduced precursor, Mia40-bound form, oxidized monomer, and assembled complex. We demonstrate that both Mia40 and Erv1 are crucial for oxidation of the precursor proteins and thus to direct vectorial transport into the IMS.

\section{MATERIALS AND METHODS}

\section{Yeast Strains}

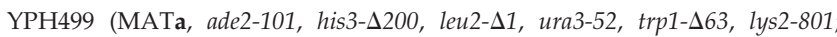
Sikorski and Hieter, 1989) was used as wild-type Saccharomyces cerevisiae strain throughout this study. The mutant strains mia40-3 (MATa, ade2-101, his3- $\Delta 200$, leu2- $\Delta 1$, ura3-52, trp1- 663 , lys2-801, mia40::ADE2 [pFL39-FOMP28ts / mia40-3], mia40-4 (MATa, ade2-101, his3- 2 200, leu2- 1 1, ura3-52, trp1- $\Delta 63$ lys2-801, mia40::ADE2 [pFL39-FOMP2-7ts/mia40-4] and erv1-2 (YBG-0702b MATa, ade2-101, his3- $\Delta 200$, leu2- $\Delta 1$, ura3-52, trp1- $\Delta 63$, lys2-801, erv1::ADE2 [pFL39-erv1-2]) were generated by error-prone polymerase chain reaction (PCR) followed by plasmid shuffling, and they were reported previously (Chacinska et al., 2004; Rissler et al., 2005). The mutation in the erv1-2 strain led to the following amino-acid change: N166D. The mutant strains erv1-1 (YPHBG-Erv1ts-F124S; MATa, ade2-101, his3- $\Delta 200$, leu2- $\Delta 1$, ura3-52, trp1- $\Delta 63$, lys2801, erv1::ADE2 [pFL39-erv1-F124S]) and erv1-5 (YPH-BG-Erv1ts-C159S; MATa, ade2-101, his3- 200 , leu2- 1 1, ura3-52, trp1- 663 , lys2-801, erv1::ADE2 [pFL39-erv1-C159S]) were generated in the YPH499 yeast genetic background by site-directed mutagenesis followed by plasmid shuffling. erv1-1 (also termed erv1-ts) causes the amino acid substitution F124S (Lisowsky, 1994) and erv1-5 the substitution C159S. The following single and double deletion strains of Tim8 and Tim13 were used: tim8s tim13 $\Delta$ (MATa, ade2-101, his3 $\Delta 200$, leu2- $\Delta 1$, ura3-52, trp1- $\Delta 63$, lys2-801 tim13s::kanMX5 tim8s::TRP1) (Frazier et al., 2003), tim8 (MATa, ade2-101, his3- $\Delta 200$, leu2- $\Delta 1$, ura3-52, trp1- $\Delta 63$, lys2-801 tim8s::TRP1) and tim13 $($ MATa, ade2-101, his3- 200 , leu2- $\Delta 1$, ura352, trp1- $\Delta 63$, lys2-801 tim13 $\Delta:: k a n M X 6)$

Yeast strains were grown at $24^{\circ} \mathrm{C}$ (temperature-sensitive mutants and corresponding YPH499 wild-type strain) and at $30^{\circ} \mathrm{C}$ for deletion mutant strains in medium containing $1 \%(\mathrm{wt} / \mathrm{vol})$ yeast extract, $2 \%$ (wt/vol) bacto-peptone, and $3 \%(\mathrm{vol} / \mathrm{vol})$ glycerol (YPG). To test the growth of yeast cells, serial 10 -fold dilutions of a liquid yeast culture were prepared, spotted on YPD (1\% (wt/vol) yeast extract, $2 \%$ (wt/vol) bacto-peptone, 2\% (wt/vol) dextrose) plates, and incubated at 24 and $37^{\circ} \mathrm{C}$.

\section{Mitochondrial Isolation}

Mutant and corresponding wild-type strains were grown in liquid YPG medium to an $\mathrm{OD}_{600 \mathrm{~nm}}$ of $\sim 1.0$. Mitochondria were isolated by differential centrifugation (Daum et al., 1982; Meisinger et al., 2006). The mitochondrial concentration was adjusted to a protein concentration of $10 \mathrm{mg} / \mathrm{ml}$ in SM buffer (250 mM sucrose and $10 \mathrm{mM}$ MOPS/KOH, pH 7.2). To check the steady-state levels of mitochondrial proteins, mitochondria were solubilized in Laemmli buffer, separated by SDS-polyacrylamide gel electrophoresis (PAGE), and analyzed by Western blotting according to standard procedures.

\section{Synthesis of Radiolabeled Precursor Proteins}

The open reading frames of TIM8 and TIM13 were cloned into a pGEM4Z vector downstream of the SP6 promotor. These plasmids were used for generation of cysteine mutants in which single cysteines were changed to serines by PCR-based site-directed mutagenesis (QuikChange; Stratagene, La Jolla, CA) according to the protocol of the manufacturer. The pGEM4ZTim8 and pGEM4Z-Tim13 plasmids and their derivatives harboring the cysteine mutants of Tim 8 and Tim13 were used for in vitro transcription using the mMESSAGE mMACHINE kit (Ambion, Austin, TX) and translation in rabbit reticulocyte lysate in the presence of [ $\left.{ }^{35} \mathrm{~S}\right]$ methionine (GE Healthcare, Little Chalfont, Buckinghamshire, United Kingdom). Synthesized proteins were precipitated with a saturated solution of ammonium sulfate and denatured in urea buffer (8 M urea, $30 \mathrm{mM}$ MOPS/KOH, pH 7.2, and $50 \mathrm{mM}$ dithiothreitol [DTT])

\section{Protein Import into Mitochondria Followed by Thiol Modification}

${ }^{35} \mathrm{~S}$-labeled precursors ( $2 \%$ vol of the import reaction) were incubated with 50-100 $\mu \mathrm{g}$ (protein amount) of isolated mitochondria at $30^{\circ} \mathrm{C}$ in import buffer 
[ $3 \%$ (wt/vol) fatty acid-free bovine serum albumin, $250 \mathrm{mM}$ sucrose, $80 \mathrm{mM}$ $\mathrm{KCl}, 5 \mathrm{mM} \mathrm{MgCl}$, $5 \mathrm{mM}$ methionine, $10 \mathrm{mM} \mathrm{KH} \mathrm{PO}_{4}$, and $10 \mathrm{mM}$ MOPS/ $\mathrm{KOH}, \mathrm{pH}$ 7.2). To stop the import reaction, samples were placed on ice. Where indicated, the samples were incubated with $50 \mu \mathrm{g} / \mathrm{ml}$ proteinase K. SEM buffer (250 mM sucrose, $1 \mathrm{mM}$ EDTA, and $10 \mathrm{mM}$ MOPS/KOH, $\mathrm{pH} 7.2$ ) was used for washing of the samples. To alkylate proteins with 4-aceto-4'-maleimidylstilbene-2,2'-disulfonic acid (AMS), mitochondria were reisolated in 20 $\mu \mathrm{l}$ of AMS buffer (15 mM AMS, $6 \mathrm{M}$ urea, $200 \mathrm{mM}$ Tris- $\mathrm{HCl}$, $\mathrm{pH}$ 8.5, and 10 mM EDTA). The samples were incubated at $25^{\circ} \mathrm{C}$ for $1 \mathrm{~h}$. To block thiols with iodoacetamide (IA), the mitochondria were reisolated and resuspended in Laemmli buffer (without reducing agent) containing $50 \mathrm{mM}$ IA, and then they were analyzed by Tricine-SDS-PAGE (Schägger and von Jagow, 1987). For native analysis, mitochondria were solubilized in digitonin solubilization buffer [1\% (wt/vol) digitonin, $20 \mathrm{mM}$ Tris-HCl, $0.5 \mathrm{mM}$ EDTA, 10\% (vol/vol) glycerol, $50 \mathrm{mM} \mathrm{NaCl}$, and $1 \mathrm{mM}$ phenylmethylsulfonyl fluoride, $\mathrm{pH} 7.4$ ) containing $50 \mathrm{mM} \mathrm{IA}$, and extracts were separated by blue native gel electrophoresis (Schägger and von Jagow, 1991; Dekker et al., 1997; Chacinska et al., 2004). The position of molecular weight markers was determined from gel lanes run under the same conditions as the lanes containing radiolabeled proteins. Gels were analyzed by digital phosphorimage autoradiography (GE Healthcare)

\section{RESULTS}

\section{Monitoring Oxidation of Small Tim Proteins during Import into Mitochondria}

We used Tim 8 and Tim13 to establish assays if the precursor proteins are oxidized or reduced upon import into mitochondria. The radiolabeled and reduced precursor of Tim13 was incubated with isolated yeast mitochondria. Mitochondria were isolated and analyzed by denaturing electrophoresis under nonreducing conditions (nonreducing SDS-PAGE) to preserve disulfide bonds. Interestingly, Tim13 migrated as a doublet upon import into isolated mitochondria (Figure 1A, lanes 3 and 4). We hypothesized that these bands might reflect different redox states of Tim13, and we used AMS to modify thiol groups. Addition of AMS to a single cysteine residue of a protein leads to a size shift corresponding to 500 Da. When Tim13 imported into mitochondria was treated with AMS, the lower band was shifted to the higher molec-

A

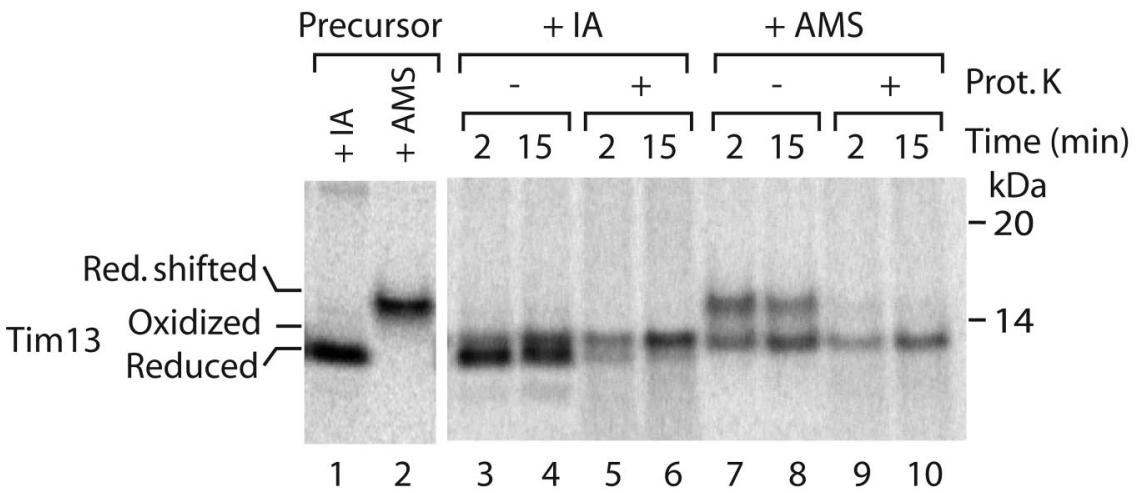

B

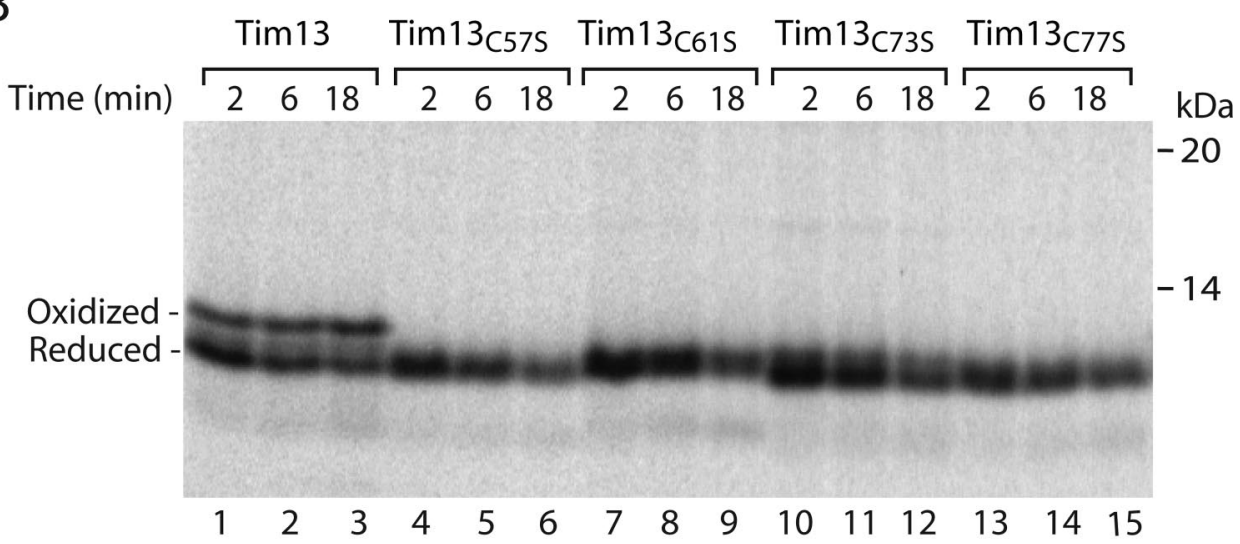

C

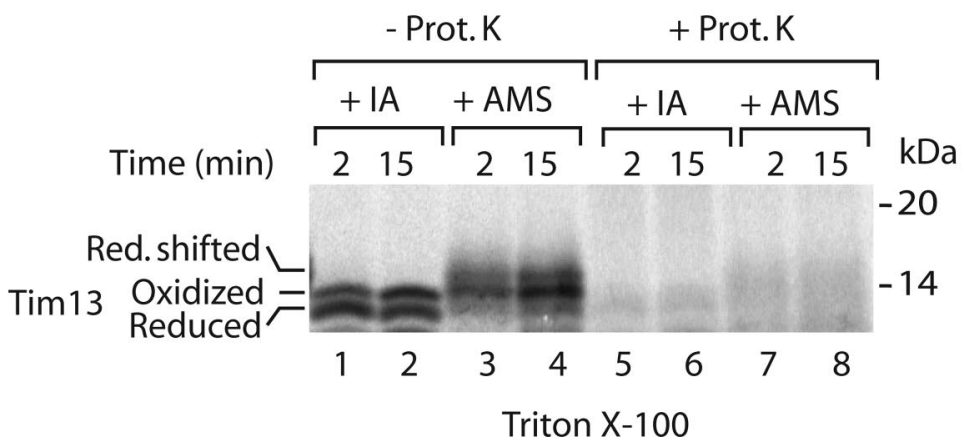

Figure 1. Tim13 is oxidized upon import into mitochondria. (A) Isolated yeast wild-type mitochondria were incubated with ${ }^{35}$ S-labeled precursor of Tim13 for the indicated times. Mitochondria were treated with proteinase K (Prot. K), reisolated, and subjected to denaturation in the presence of $50 \mathrm{mM}$ IA or $15 \mathrm{mM}$ AMS as indicated. Lanes 1 and 2, the Tim13 precursor was directly treated with $50 \mathrm{mM} \mathrm{IA}$ or $15 \mathrm{mM}$ AMS as indicated to serve as a migration standard. Red., reduced. (B) ${ }^{35}$ S-labeled wildtype Tim13 and single cysteine mutants of Tim 13 were incubated with wild-type mitochondria for the indicated times and analyzed without proteinase $\mathrm{K}$ treatment. When the mitochondria were treated with proteinase $\mathrm{K}$ after the import reaction, the single cysteine mutants were accessible to the protease to the same extent as the reduced wild-type precursor of Tim13. (C) ${ }^{35}$ S-labeled Tim 13 was imported for the indicated time periods. Proteinase $\mathrm{K}$ treatment was performed in SEM buffer containing 1\% Triton X-100. Proteins were precipitated before IA or AMS treatment. Samples were analyzed by nonreducing Tricine-SDS-PAGE. Protein bands were visualized by digital autoradiography. 
ular weight range (Figure 1A, lanes 7 and 8). This shift corresponded to the size shift that was observed when all four cysteines of the free, reduced Tim13 precursor were modified with AMS (Figure 1A, lanes 1 and 2). Thus, the lower band represented the reduced form. The upper band, which was accumulated upon incubation with mitochondria, remained unchanged upon AMS modification (Figure 1A), suggesting that it may contain disulfides. To substantiate this finding, we constructed four mutant forms of Tim13 by replacing each cysteine residue with serine individually. The cysteine mutants of Tim13 were imported into mitochondria and analyzed by nonreducing SDS-PAGE. Neither of them formed the upper band (Figure 1B). Thus, the upper band represents an oxidized form of Tim13. Gen- eration of this form required all four cysteine residues of the precursor.

Oxidized, but not reduced, Tim13 was protected against proteinase $\mathrm{K}$ added to mitochondria (Figure 1A, lanes 5, 6, 9, and 10). To eliminate the possibility of an intrinsic proteinase $\mathrm{K}$ resistance of the oxidized protein, mitochondria were solubilized by Triton X-100. Now, also the oxidized form of Tim13 was efficiently digested by proteinase K (Figure 1C). We conclude that oxidized Tim13 formed upon incubation with mitochondria was transported to a protease-protected location.

Radiolabeled Tim8 imported into mitochondria migrated as a single band on nonreducing SDS-PAGE (Figure 2A, lanes 3 and 4 ) in contrast to Tim13, but similar to Tim9 and

A

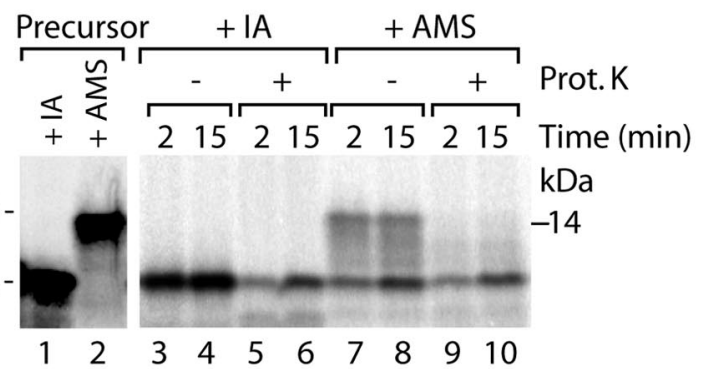

B

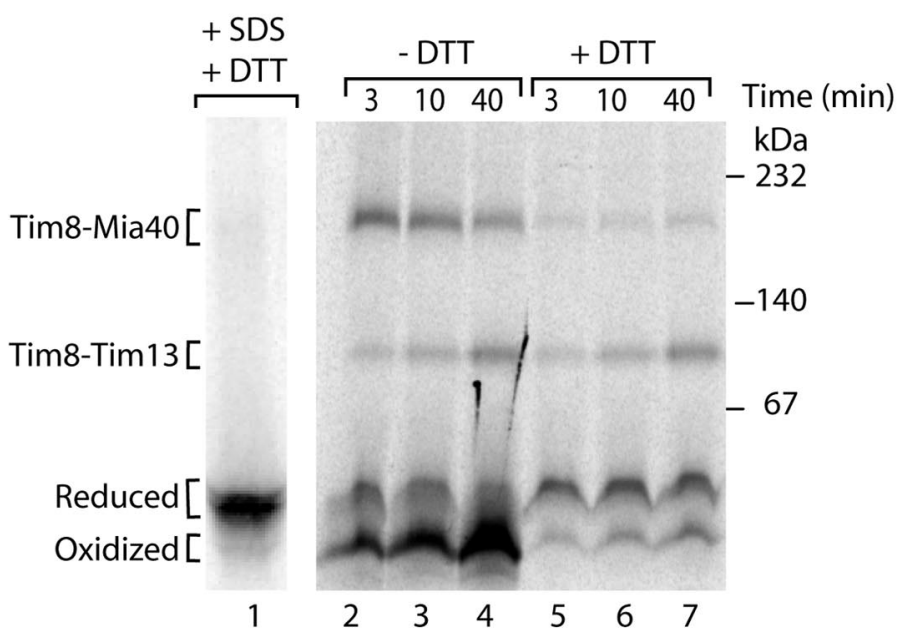

Figure 2. Tim8 is oxidized upon import into mitochondria. (A) Isolated wild-type mitochondria were incubated with ${ }^{35}$ S-labeled precursor of Tim8 for the indicated times. Mitochondria were treated with proteinase $\mathrm{K}$ (Prot. K), reisolated, and subjected to denaturation in the presence of $50 \mathrm{mM}$ IA or 15 $\mathrm{mM}$ AMS. The samples were analyzed by nonreducing Tricine-SDS-PAGE. Lanes 1 and 2 , the Tim8 precursor was directly treated with 50 mM IA or 15 mM AMS. Oxid. + Red., oxidized and reduced. (B and C) ${ }^{35}$ S-labeled precursors of Tim 8 were imported into isolated yeast wild-type mitochondria. In B, mitochondria were reisolated and treated with $50 \mathrm{mM}$ DTT for $15 \mathrm{~min}$ at $20^{\circ} \mathrm{C}$ as indicated; lane 1 , import was performed for $30 \mathrm{~min}$, and mitochondria were solubilized in the presence of $0.5 \%$ SDS and heated at $75^{\circ} \mathrm{C}$. All samples of $\mathrm{B}$ and $\mathrm{C}$, after solubilization in the presence of $50 \mathrm{mM}$ IA, assembly reactions were separated by blue native electrophoresis and analyzed by digital autoradiography.

C Tim8 Tim8C44S Tim8C48S Tim8C64S Tim8C68S

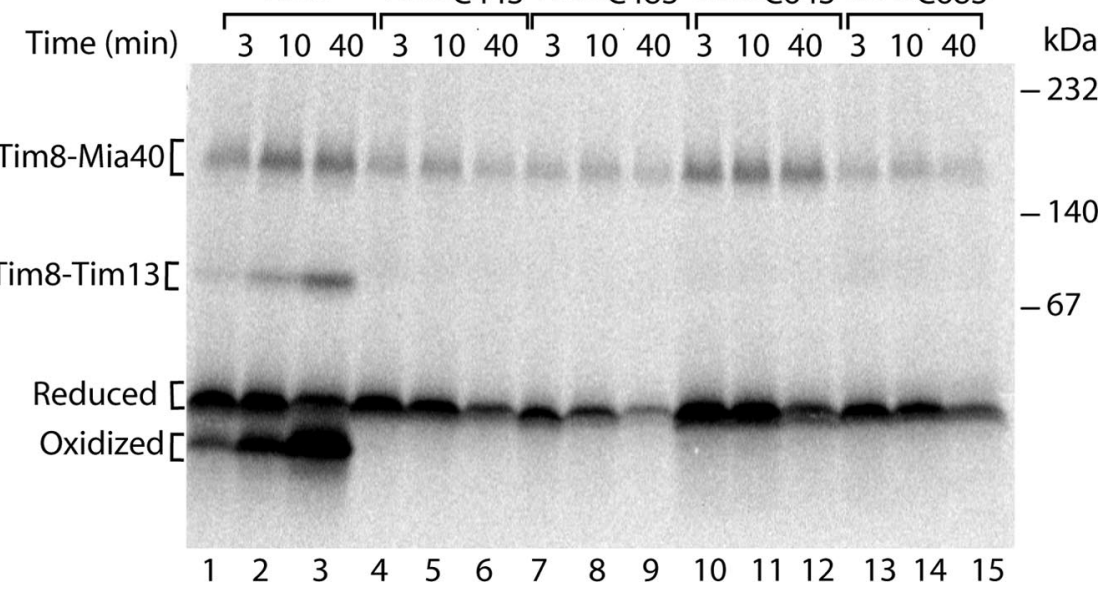


Tim10 (Milenkovic et al., 2007a). When the mitochondria were treated with AMS, however, a fraction of Tim8 exhibited a slower mobility during electrophoresis (Figure 2A, lanes 7 and 8) in agreement with the size shift observed for the modification of all four cysteine residues present in the free, reduced precursor (Figure 2A, lanes 1 and 2). The reduced form was sensitive to proteinase $\mathrm{K}$ added to mitochondria (Figure 2A, lanes 9 and 10), demonstrating that it had not crossed the outer mitochondrial membrane. Similar to Tim13, the oxidized fraction of Tim8, which was resistant to AMS modification, was protected against externally added proteinase $\mathrm{K}$ (Figure $2 \mathrm{~A}$, lanes 9 and 10).

Blue native electrophoresis of digitonin-lysed mitochondria dissects the assembly pathway of radiolabeled Tim8 in the early formation of a Mia40 intermediate and the subsequent generation of the mature Tim8-Tim13 complex (Chacinska et al., 2004). Remarkably, when the low-molecular-weight region of the gradient gel was analyzed, we detected two distinct bands (Figure 2B, lanes 2-4). We hypothesized that these bands may reflect the reduced and oxidized monomeric forms of Tim8. To test this possibility, mitochondria were treated with the reducing agent DTT after the import of Tim8 (Figure 2B, lanes 5-7). Thereby, the amounts of the disulfide conjugated Tim8-Mia40 intermediate and of the lowest band were strongly lowered. The mature Tim8Tim13 complex remained unaffected in agreement with the previously reported resistance of the fully assembled complexes to reductant treatment (Curran et al., 2002b). The second lowest band also remained after the treatment with DTT (Figure 2B, lanes 5-7). The mobility of this band was not changed by a harsh treatment upon heating in SDS (Figure 2B, lane 1), indicating that it contained the reduced monomeric form. We conclude that the two low-molecularweight bands represent the reduced and oxidized monomeric forms of Tim8, respectively.

To obtain further evidence for the presence of an oxidized monomeric form of Tim8, we generated precursors where the individual cysteine residues were replaced with serine. We tested the ability of the mutant precursors to assemble into the native complexes after import into mitochondria.
Each cysteine residue was essential for generation of the mature Tim8-Tim13 complex (Figure 2C) in agreement with the role of cysteine residues in the biogenesis of Tim 9 and Tim10 (Allen et al., 2003; Lu et al., 2004a; Milenkovic et al., 2007a; Sideris and Tokatlidis, 2007). Importantly, none of the mutant precursors generated the faster migrating low-molecular-weight band (Figure 2C). Thus, each cysteine residue is required to generate this band, supporting the conclusion that it represents an oxidized monomer of Tim8.

Together, the native assay separates four different pools of Tim8: reduced precursor, Mia40-bound species, oxidized monomer, and mature oligomeric complex. This assay represents the first demonstration of an oxidized monomeric form of a small Tim protein generated during import into mitochondria.

\section{The Oxidized Monomer of Tim8 Is Retained in the IMS}

We used proteinase $\mathrm{K}$ to probe for the localization of the two monomeric forms of Tim8. The reduced monomer was digested by the protease (Figure $3 \mathrm{~A}$ ), indicating that it was not protected by the mitochondrial membranes. In contrast, oxidized monomeric Tim8 was fully protected against proteolytic attack like the assembled Tim8-Tim13 chaperone complex (Figure 3A), demonstrating that it was located inside the mitochondria.

We noticed that upon treatment of mitochondria with DTT, the total radiolabeled signal of the four forms of Tim8 together was significantly lower than in the absence of DTT (Figure 2B). Because after the DTT treatment the mitochondria were pelleted by centrifugation before applying them to blue native gels, we asked whether precursor was released to the supernatant. Indeed, we observed a large amount of reduced monomeric Tim8 in the supernatant (Figure 3B, lanes 7 and 8 ) that reflected well the overall loss of signal in the mitochondrial pellet (Figure 3B, lanes 3 and 4). The hexameric Tim8-Tim13 complex and the residual amount of Mia40-bound Tim8 were not released to the supernatant (Figure 3B, lanes 7 and 8), demonstrating that the mitochondrial outer membrane barrier remained intact upon the treatment with DTT. In the absence of DTT treatment, only
A

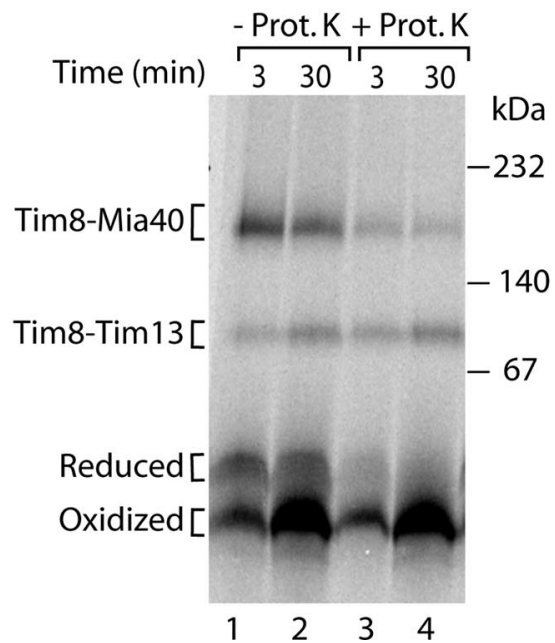

B

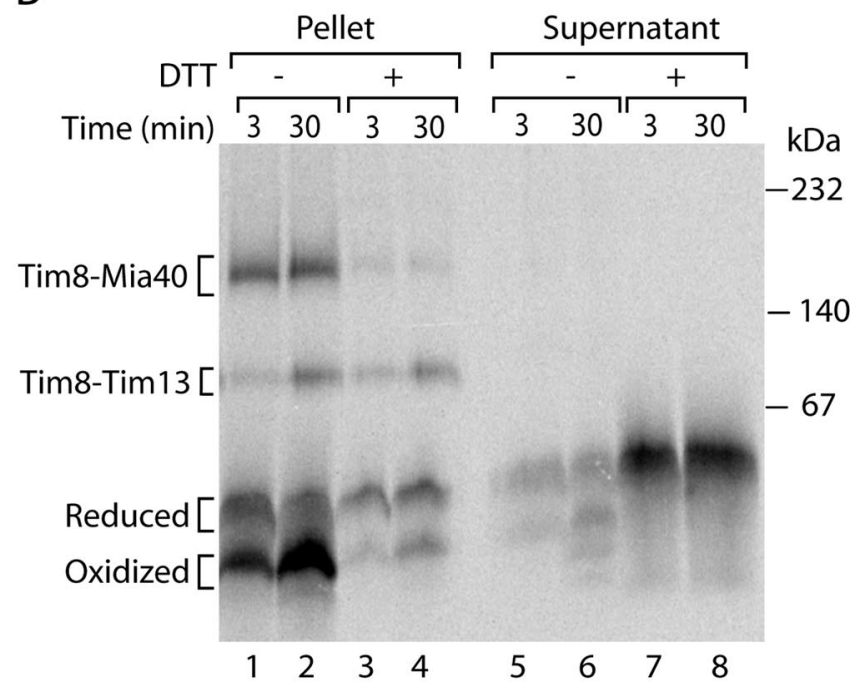

Figure 3. The oxidized monomer of Tim 8 is located inside mitochondria and released after reduction. ${ }^{35} \mathrm{~S}-\mathrm{labeled}$ precursors of Tim8 were imported into isolated yeast wild-type mitochondria. (A) Mitochondria were reisolated and treated with proteinase $\mathrm{K}$ (Prot. K) as indicated. (B) Mitochondria were spun down, and supernatants were collected. Half of the samples were treated with $50 \mathrm{mM}$ DTT at $20^{\circ} \mathrm{C}$ for $15 \mathrm{~min}$. Mitochondrial pellets and supernatants were analyzed by blue native electrophoresis and autoradiography. 
A

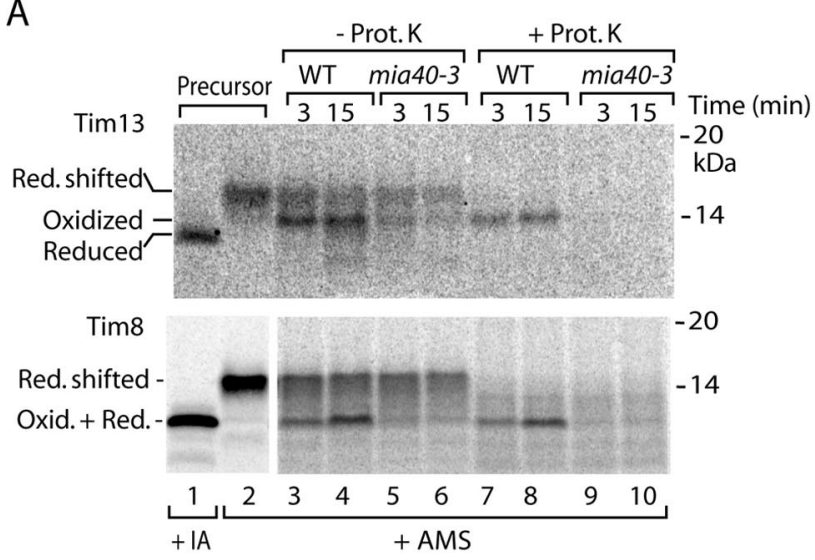

C

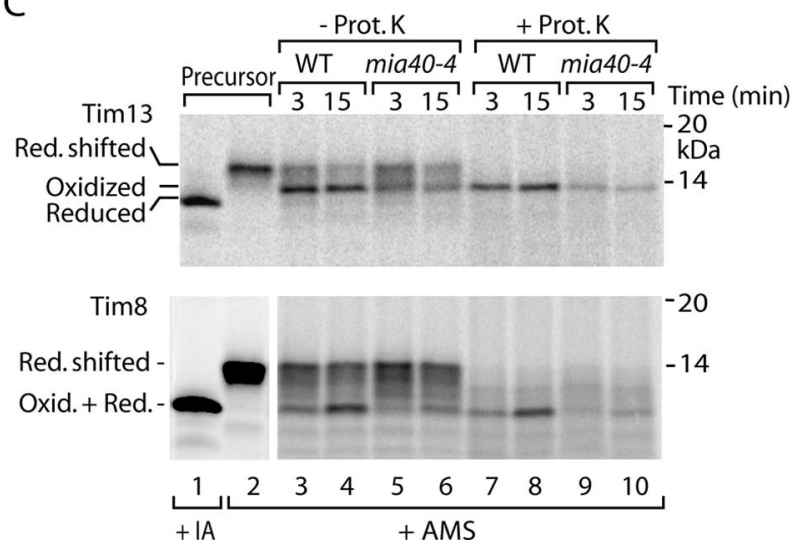

B

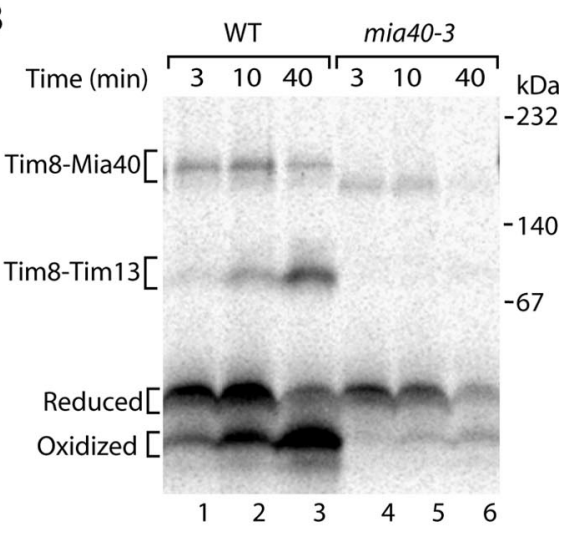

D

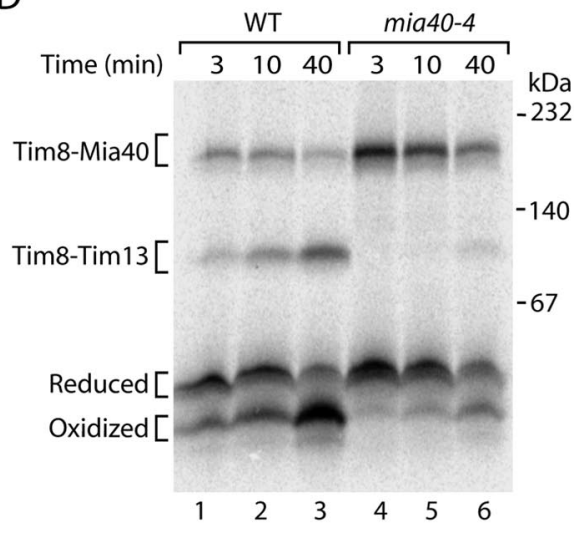

Figure 4. Mia40 is required to oxidize small Tim proteins. (A and C) ${ }^{35}$ S-labeled precursors of Tim13 and Tim8 were imported into wild-type (WT) and mia40 mutant mitochondria. Where indicated, the mitochondria were treated with proteinase K (Prot. K.) followed by denaturation in the presence of $15 \mathrm{mM}$ AMS. Oxid., oxidized; Red., reduced. Lanes 1 and 2, precursors were directly treated with $50 \mathrm{mM}$ IA or $15 \mathrm{mM}$ AMS. Proteins were separated by nonreducing Tricine-SDS-PAGE and analyzed by autoradiography. (B and D) Import of ${ }^{35}$ S-labeled Tim8 into WT and mia40 mutant mitochondria was performed for the indicated times and analyzed by blue native electrophoresis.

minute amounts of the large amount of oxidized monomer were found in the supernatant (Figure 3B, lanes 1 and 2 vs. lanes 5 and 6). We conclude that the oxidized monomer is largely retained inside mitochondria. On treatment with reductant, Tim 8 is reduced and the reduced monomer is efficiently released into the supernatant.

\section{Oxidation of Small Tim Proteins Requires Functional Mia40}

Analysis of the time dependence of Tim8 import indicated that formation of the oxidized monomer was slower than formation of the Tim8-Mia40 intermediate (Figure 2B), suggesting that the oxidized monomer was generated after the interaction with Mia40. To directly address if Mia40 was required for precursor oxidation, we used two yeast mutants of Mia40. In the mia40-3 mutant mitochondria, the interaction of Mia40 with the incoming precursors is impaired (Chacinska et al., 2004). When the precursors of Tim13 and Tim8 were incubated with mia40-3 mutant mitochondria, the formation of the oxidized forms was strongly inhibited (Figure 4A). The blue native analysis of Tim8 assembly demonstrated that the mia40-3 mutant mitochondria were strongly impaired in formation of the oxidized monomer of Tim 8 and of the mature Tim8-Tim13 complex (Figure 4B). In mia40-4 mutant mitochondria, incoming precursors are accumulated at the Mia40 stage (Chacinska et al., 2004; Milenkovic et al., 2007a). Oxidation of Tim13 and Tim8 was again inhibited in the mutant mitochondria (Figure 4C). The native assay showed that formation of the oxidized Tim8 monomer was impaired (Figure 4D), whereas the amount of Mia40 intermediate was increased as expected (Chacinska et al., 2004). Thus, both mia40 mutants indicate that functional Mia40 is required for oxidation of the small Tim precursors, including formation of the oxidized monomer.

\section{Erv1 Is Required for Oxidation of Small Tim Proteins}

Allen et al. (2005) reported that small Tim proteins were still oxidized in the original yeast mutant of Erv1 (erv1-1) (Lisowsky, 1994), raising the question whether oxidation of the precursors depended on functional Erv1. To test this with our assay, we generated the erv1-1 mutant, i.e., replacing phenylalanine 124 of Erv1 with serine, in our yeast genetic background. The resulting mutant strain was temperature-sensitive as reported previously (Lisowsky, 1994). We imported radiolabeled Tim 8 and Tim13 into isolated erv1-1 mitochondria and observed that formation of the oxidized forms was only slightly impaired (Figure 5A) in agreement with the findings by Allen et al. (2005). We envisaged two possibilities. Either Erv1 did not play a critical role in oxidation of small Tim precursors or the mutant erv1-1 still retained an oxidative capacity. We thus used a 
A

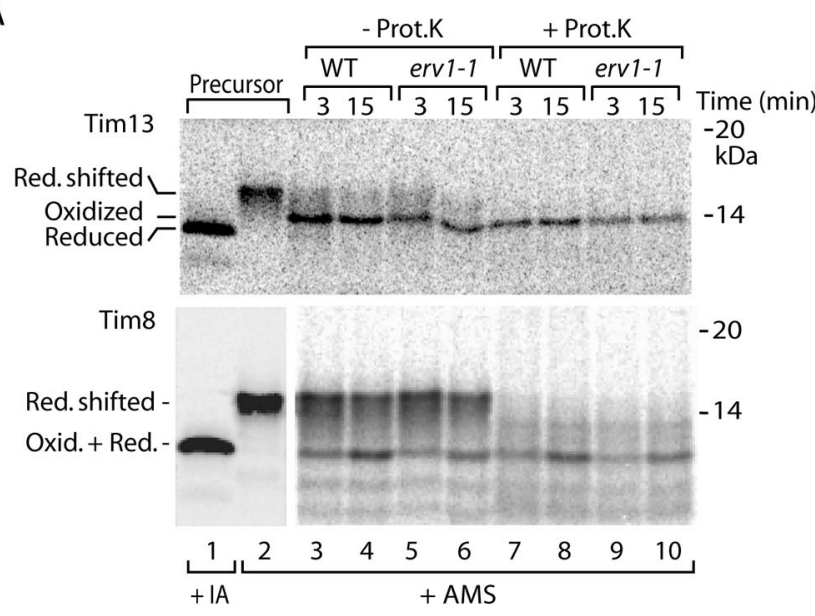

B

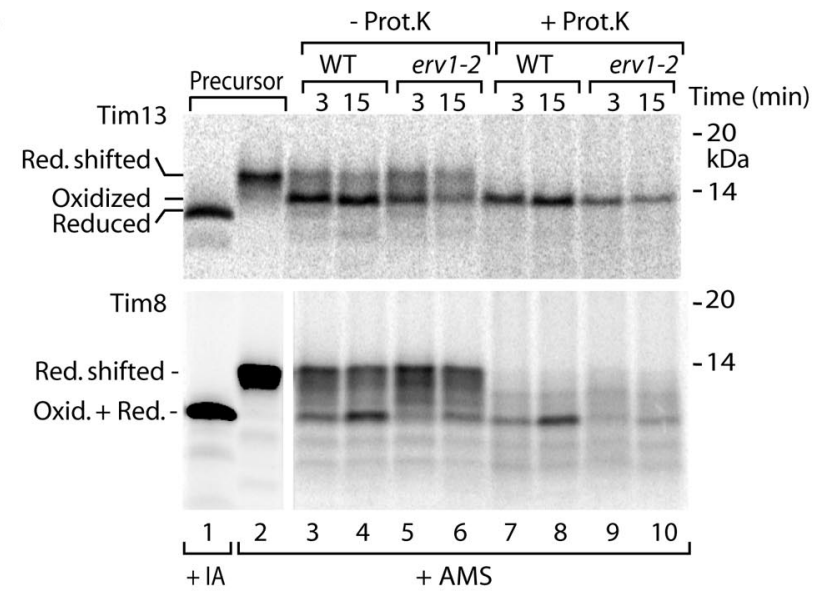

C

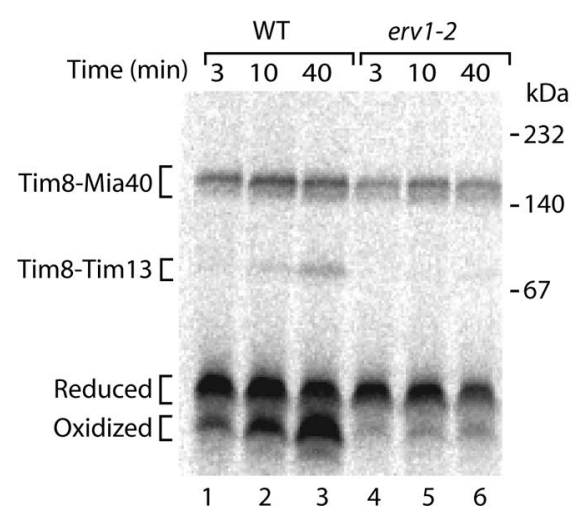

Figure 5. Influence of erv1 mutants on oxidation of small Tim proteins. (A and B) ${ }^{35} \mathrm{~S}$-labeled precursors of Tim13 and Tim8 were imported in wild-type (WT) and erv1 mutant mitochondria. Where indicated, the mitochondria were treated with proteinase $\mathrm{K}$ (Prot. K.), followed by denaturation in the presence of $15 \mathrm{mM}$ AMS. Lanes 1 and 2, precursors were directly treated with $50 \mathrm{mM}$ IA or $15 \mathrm{mM}$ AMS. Proteins were separated by nonreducing Tricine-SDS-PAGE and analyzed by autoradiography. (C) Import of ${ }^{35}$ S-labeled Tim8 into WT and erv1-2 mutant mitochondria for the indicated time periods, followed by blue native electrophoresis.

further yeast mutant, erv1-2 (Rissler et al., 2005), and we determined that a different amino acid residue of Erv1 was replaced (asparagine 166 with aspartic acid). The mutant mitochondria were partially inhibited in oxidation of Tim13
(Figure 5B, top) and strongly inhibited in oxidation of Tim8 (Figure 5B, bottom, and C).

Because these results did not reveal a full answer on the role of Erv1 in precursor oxidation, we generated a further ero1 yeast mutant by site-directed replacement of the codon for cysteine 159 with serine and termed the mutant erv1-5 (Figure 6A). This cysteine residue is required for stabilizing the FAD binding site of Erv1 (Hofhaus et al., 2003). erv1-5 mutant cells were temperature-sensitive lethal for growth (Figure 6B). We thus grew the cells at low temperature. The steady-state levels of various proteins tested, including translocase components of the outer membrane, IMS and inner membrane, were present in similar amounts in erv1-5 mitochondria and wild-type mitochondria (Figure 6C). Thus, the isolated mitochondria showed a normal mitochondrial protein composition, excluding unspecific pleiotropic effects of the mutants grown at low temperature. Previous studies with various translocase mutants showed that under these conditions kinetic assays with radiolabeled precursors often revealed the primary function of a component (Truscott et al., 2002; Chacinska et al., 2004, 2005; Meisinger et al., 2004, 2007; Rissler et al., 2005). We first used the standard assay for the MIA-system, assembly of the precursor of Tim9 (Chacinska et al., 2004), and we found that the Mia40 intermediate was still formed but that generation of the mature Tim9Tim10 and TIM22 complexes was blocked in erv1-5 mitochondria (Figure 6D). Similarly, the precursor of Tim13 still bound to Mia40, but it did not form the mature Tim8-Tim13 complex (Figure 6E). Oxidation of the precursors of Tim13 (Figure 6F) and Tim8 (Figure 6G) was severely inhibited in erv1-5 mitochondria. The native analysis demonstrated that the formation of oxidized Tim8 monomer was strongly inhibited, whereas Tim8 still efficiently bound to Mia40 (Figure $6 \mathrm{H}$ ). We conclude that functional Erv1 is required for oxidation of small Tim proteins and that this event occurs after precursor interaction with Mia40.

\section{The Oxidized Tim8 Monomer Is a Productive Intermediate in Formation of the Tim8-Tim13 Complex}

We asked whether the oxidized monomeric form of Tim8 represented an intermediate in formation of the Tim8Tim13 complex. To address this question, we used yeast deletion mutants lacking either Tim8 or Tim13 or both (Figure 7A, lanes 1-4) (Truscott et al., 2002; Frazier et al., 2003; Wiedemann et al., 2004). The steady-state levels of translocase subunits from different mitochondrial subcompartments were similar in the mutant mitochondria and wildtype mitochondria (Figure 7A, lanes 5-12). Oxidation of the radiolabeled precursor of Tim13 was examined by import into mitochondria, followed by treatment with proteinase $\mathrm{K}$ and nonreducing SDS-PAGE. The mutant mitochondria oxidized Tim13, with an efficiency comparable with wild-type mitochondria (Figure 7B). Thus, the lack of the partner protein Tim8 in tim $8 \Delta$ and tim $8 \Delta$ tim $13 \Delta$ mitochondria (and in consequence the lack of formation of the mature Tim8Tim13 complex) did not inhibit the acquisition of disulfide bonds by Tim13, suggesting that complex formation was not a prerequisite for oxidation of Tim13.

The import of Tim 8 was analyzed by blue native electrophoresis. The Tim8-Mia40 intermediate was formed with similar efficiency in mutant and wild-type mitochondria (Figure 7C). As expected, Tim8 could not be assembled into a mature Tim8-Tim13 complex in tim $13 \Delta$ mitochondria and $\operatorname{tim} 8 \Delta \operatorname{tim} 13 \Delta$ mitochondria. However, the assembly of Tim8 in tim $8 \Delta$ mitochondria proceeded much faster than in wildtype mitochondria (Figure $7 \mathrm{C}$, lanes $4-6$ vs. lanes $7-9$ ). This result was surprising, because immunodecoration showed 
A

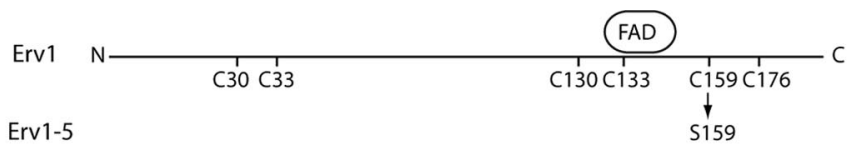

B

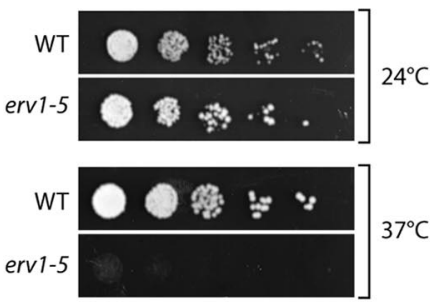

$\mathrm{D}$

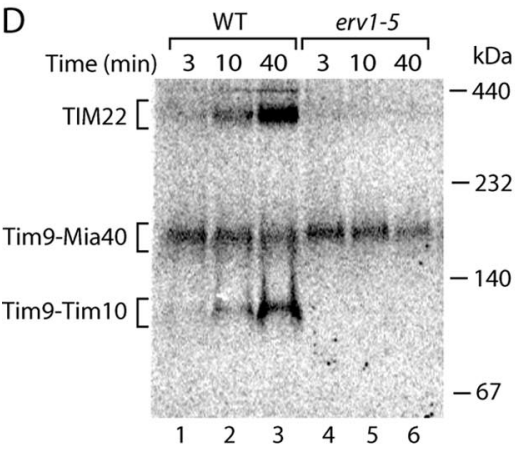

C

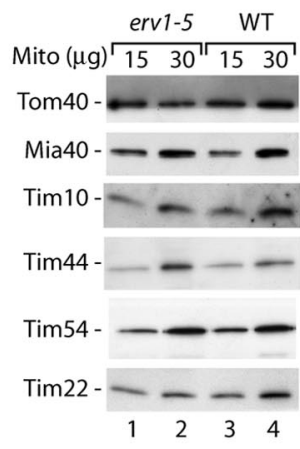

$E$

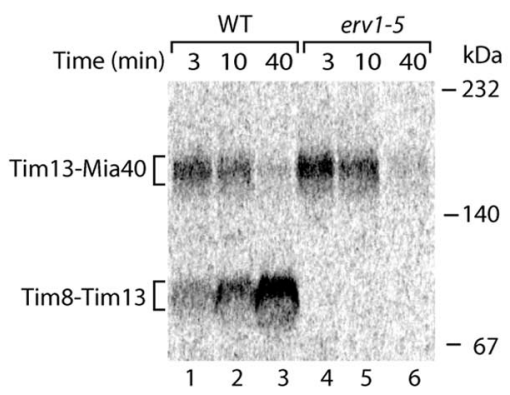

G
$\mathrm{F}$
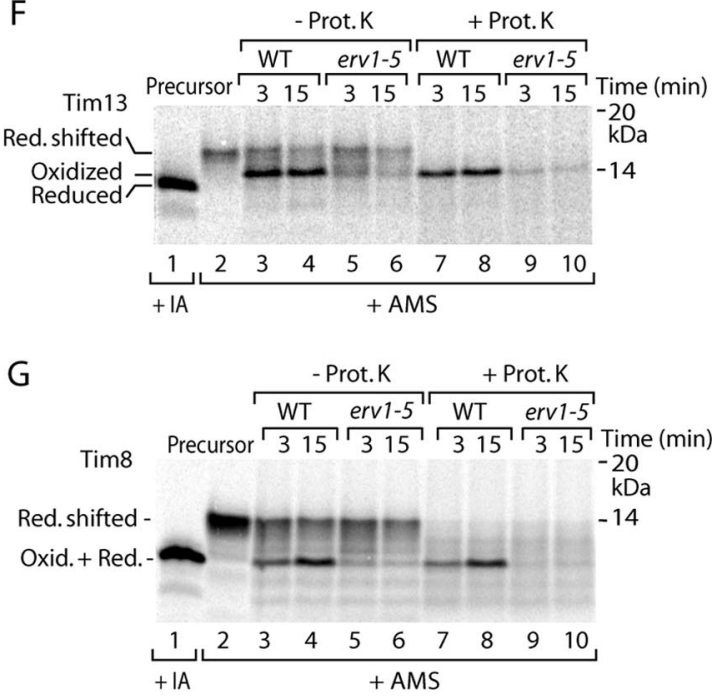

$\mathrm{H}$

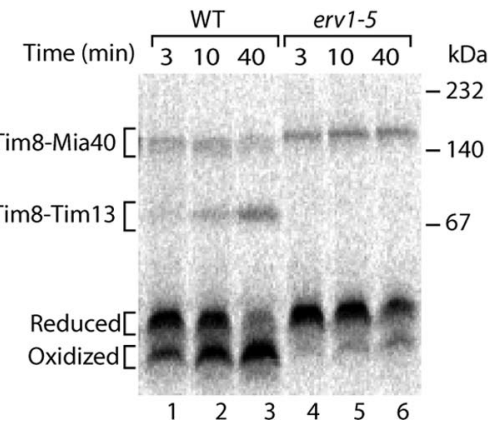

Figure 6. Cysteine 159 mutant of Erv1 (erv1-5) is defective in formation of disulfide bonds in small Tim proteins. (A) Schematic representation of Erv1. The cysteine residues and FAD binding site in the primary structure of S. cerevisiae Erv1 are indicated previously (Hofhaus et al., 2003). Erv1-5 contains the amino acid substitution C159S. (B) Wild-type (WT) and erv1-5 cells were subjected to serial dilutions, plated on YPD medium and incubated at the indicated temperatures. (C) Mitochondria (15 and $30 \mu \mathrm{g}$ of protein) from WT and erv1-5 strains were analyzed by SDS-PAGE and Western blotting. (D and E) ${ }^{35}$ S-labeled precursors of Tim9 and Tim13, respectively, were incubated with WT and erv1-5 mitochondria. The mitochondria were solubilized with digitonin and analyzed by blue native electrophoresis and autoradiography. (F and G) ${ }^{35}$ S-labeled precursors of Tim13 and Tim8 were imported into WT and erv1-5 mutant mitochondria. Where indicated, the mitochondria were treated with proteinase K (Prot. K.), followed by denaturation in the presence of $15 \mathrm{mM}$ AMS. Lanes 1 and 2, precursors were directly treated with $50 \mathrm{mM}$ IA or $15 \mathrm{mM}$ AMS. Proteins were separated by nonreducing Tricine-SDS-PAGE and analyzed by autoradiography. (H) Import of ${ }^{35}$ S-labeled Tim8 was analyzed as described for D and E.

that the lack of one partner, either Tim8 or Tim13, led to the loss of the other partner on a steady-state level (Koehler et al., 1999; Davis et al., 2000) (Figure 7A, lanes 3 and 4). We thus examined the steady-state levels of Tim 8 and Tim13 in the mutant mitochondria in more detail. Whereas both proteins were completely absent in the double mutant tim $8 \Delta$ $\operatorname{tim} 13 \Delta$ as expected, the single mutants still contained small but detectable amounts of the other partner protein (Figure 7A, lanes 3 and 4, longer exposure). In wild-type mitochondria the assembly of an individual radiolabeled subunit into a hetero-oligomeric complex requires the exchange with preexisting subunits of the complex or the association with nonassembled subunits (Dekker et al., 1997; Rapaport et al., 2001; Model et al., 2001). Because the pool of nonassembled subunits in wild-type mitochondria is very low, the yield of integration of a newly imported subunit into the complex is low. When one partner of the Tim8-Tim13 complex is lacking, however, no mature complexes exist and the remaining amounts of the other partner protein form a pool of nonassembled subunits that can rapidly associate with the incoming radiolabeled precursor to mature hexameric complexes.
Remarkably, under these conditions an efficient shift of the oxidized Tim8 monomer to the mature Tim8-Tim13 complex was observed (Figure 7C, lanes 4-6). Thus, the formation of oxidized Tim 8 precedes the assembly into the mature complexes. We conclude that the oxidized monomer is a productive intermediate on the pathway to the Tim8-Tim13 chaperone complex.

\section{DISCUSSION}

We have dissected the import and assembly pathway of mitochondrial IMS proteins by a combination of native analysis and thiol-trapping techniques. Four functional stages could be directly visualized: the reduced precursor protein, the precursor bound to Mia40, the oxidized monomer and finally the mature, assembled complex in the IMS. We detected an oxidized monomeric form of the precursor of Tim8 in organello that is formed after the interaction with Mia40 but before the assembly into the hexameric Tim8-Tim13 complex, thus representing a true intermediate in the IMS assembly pathway. 
A
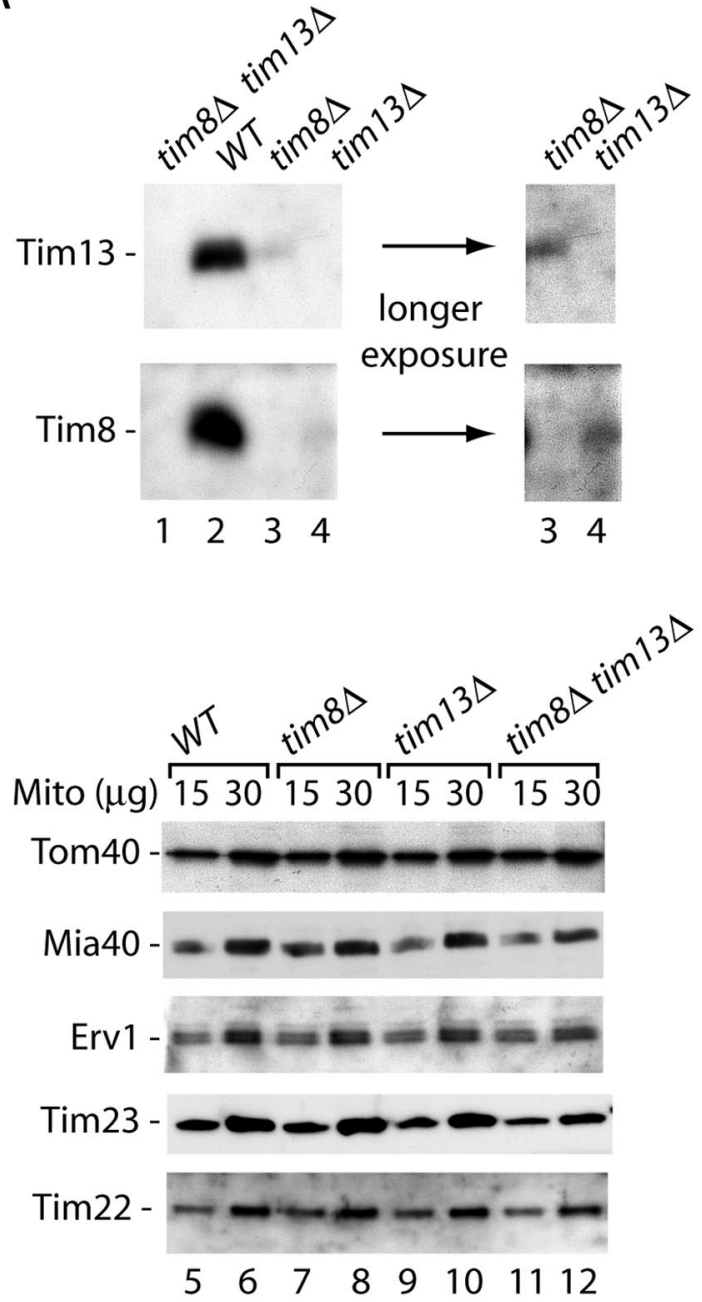

B
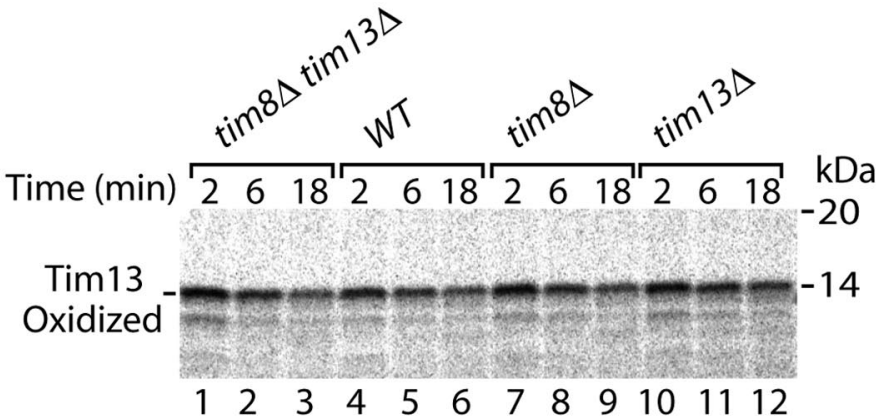

C

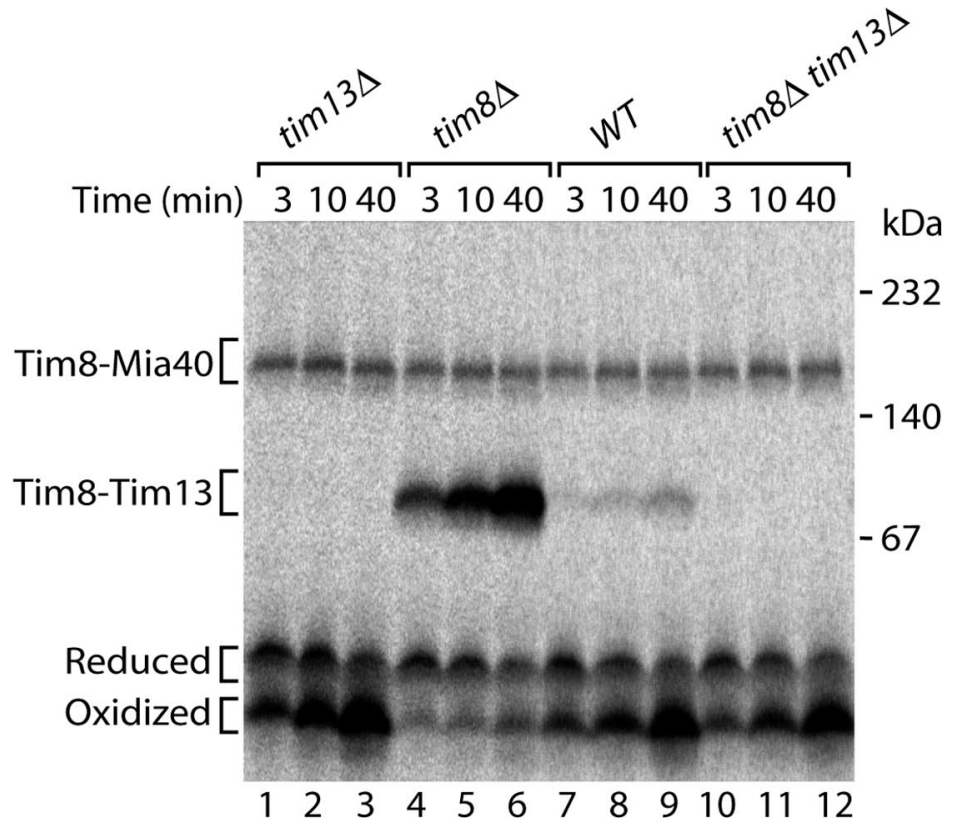

Figure 7. The oxidized monomer of Tim8 is a productive intermediate in formation of the Tim8-Tim13 complex. (A) Mitochondria from single deletion mutant strains ( $\operatorname{tim} 8 \Delta, \operatorname{tim} 13 \Delta$ ), the double deletion strain tim $8 \Delta \operatorname{tim} 13 \Delta$, and wild-type (WT) were analyzed by SDS-PAGE and Western blotting. The level of Tim8 in tim $13 \Delta$ mitochondria and the level of Tim13 in tim $8 \Delta$ mitochondria was $<5-10 \%$ of the level in wild-type mitochondria, respectively. (B and C) ${ }^{35}$ S-labeled precursors of Tim13 and Tim8, respectively, were imported into WT, tim $8 \Delta$, $\operatorname{tim} 13 \Delta$, and tim8s tim $13 \Delta$ mitochondria. In B, mitochondria were treated with proteinase $\mathrm{K}$ before nonreducing Tricine-SDS-PAGE. In C, mitochondria were solubilized in the presence of $50 \mathrm{mM}$ IA, and proteins were separated by blue native electrophoresis. Protein bands were visualized by digital autoradiography.

The identification of the oxidized monomer in mitochondria represents a critical experimental evidence that has been missing so far to demonstrate the disulfide relay hypothesis (Tokatlidis, 2005; Herrmann and Köhl, 2007; Milenkovic et al., 2007b). Transient intermolecular disulfide links between Mia40 and the sulfhydryl oxidase Erv1and between Mia40 and precursors were reported previously (Chacinska et al., 2004; Mesecke et al., 2005; Rissler et al., 2005; Milenkovic et al., 2007a; Sideris and Tokatlidis, 2007); yet, a role of Erv1 and Mia40 in oxidation of imported precursors proteins, i.e., generation of intramolecular disulfides, was not demonstrated so far. In fact, analysis of the original erv1-1 yeast mutant revealed that precursor oxidation was not impaired in vivo (Allen et al., 2005). Although we confirmed that this erv1-1 mutation indeed did not significantly inhibit precursor oxidation in the IMS, we found a defect in precursor oxidation with two different mutants of Erv1 where other amino acid residues of the sulfhydryl oxidase were replaced. Similarly, two mutants of Mia40 revealed a requirement of Mia40 for precursor oxidation. Thus, both es- sential subunits of the MIA-system are needed for oxidation of precursor proteins. Different views were also discussed with regard to the biogenesis steps that involve Erv1 (Allen et al., 2005; Mesecke et al., 2005; Rissler et al., 2005). With the resolution of distinct stages, we found that precursor binding to Mia40 still took place in the erv1 mutants, whereas the subsequent precursor release and formation of the oxidized monomer depended on active Erv1. The disulfide exchange between the MIA components ultimately results in the transfer of disulfide bonds into the imported precursor proteins. Together with the findings of Farrell and Thorpe (2005) and Allen et al. (2005) that cytochrome $c$ functions as electron acceptor for Erv1, the mitochondrial disulfide relay likely comprises the following elements: respiratory chain - cytochrome $c$ - Erv1 - Mia40 - precursor proteins.

Different models have been discussed of how the precursors of small IMS proteins are accumulated in mitochondria and prevented from diffusion into the cytosol. It was proposed that either binding of zinc ions to reduced imported precursors or oxidation of imported precursors prevented a 
retrograde movement (Lutz et al., 2003; Lu et al., 2004a); yet, an experimental clarification was not possible. Because our native assay allowed a direct monitoring of the formation and location of both reduced and oxidized monomers, we followed the appearance of the precursors in mitochondria and the postmitochondrial supernatant. The oxidized monomer, but not the reduced monomer, was fully protected against protease added to mitochondria, i.e., was located inside mitochondria. On treatment with reductant, the oxidized precursor was reduced and efficiently released to the supernatant. Thus, the oxidation of imported IMS precursors blocks their retrograde movement across the outer mitochondrial membrane.

The following model is suggested. The Erv1-Mia40 relay transfers disulfide bonds into imported precursors of small IMS proteins. Oxidation of the precursors is mechanistically coupled to their accumulation in the IMS. Thereby, Mia40 and Erv1 control the vectorial translocation of these precursor proteins into mitochondria. The oxidized monomers represent then the building blocks for assembly of the mature TIM chaperone complexes in the IMS.

\section{ACKNOWLEDGMENTS}

We thank Drs. Peter Rehling, Nils Wiedemann, and Diana Stojanovski for strains and discussion. This work was supported by the Deutsche Forschungsgemeinschaft, Sonderforschungsbereich 746, Gottfried Wilhelm Leibniz Program, Max Planck Research Award, Bundesministerium für Bildung und Forschung, and the Fonds der Chemischen Industrie.

\section{REFERENCES}

Adam, A., Endres, M., Sirrenberg, C., Lottspeich, F., Neupert, W., and Brunner, M. (1999). Tim9 a new component of the TIM22-54 translocase in mitochondria. EMBO J. 18, 313-319.

Allen, S., Balabanidou, V., Sideris, D. P., Lisowsky, T., and Tokatlidis, K. (2005). Erv1 mediates the Mia40-dependent protein import pathway and provides a functional link to the respiratory chain by shuttling electrons to cytochrome $c$. J. Mol. Biol. 353, 937-944.

Allen, S., Lu, H., Thornton, D., and Tokatlidis, K. (2003). Juxtaposition of the two distal $\mathrm{Cx}_{3} \mathrm{C}$ motifs via intrachain disulfide bonding is essential for the folding of Tim10. J. Biol. Chem. 278, 38505-38513.

Bohnert, M., Pfanner, N., and van der Laan, M. (2007). A dynamic machinery for import of mitochondrial precursor proteins. FEBS Lett. 581, 2802-2810.

Chacinska, A. et al. (2005). Mitochondrial presequence translocase: switching between TOM tethering and motor recruitment involves Tim21 and Tim17. Cell 120, 817-829.

Chacinska, A., Pfannschmidt, S., Wiedemann, N., Kozjak, V., Sanjuán Szklarz, L. K., Schulze-Specking, A., Truscott, K. N., Guiard, B., Meisinger, C., and Pfanner, N. (2004). Essential role of Mia40 in import and assembly of mitochondrial intermembrane space proteins. EMBO J. 23, 3735-3746.

Collet, J. F., and Bardwell, J. C. (2002). Oxidative protein folding in bacteria. Mol. Microbiol. 44, 1-8.

Curran, S. P., Leuenberger, D., Oppliger, W., and Koehler, C. M. (2002a). The Tim9p-Tim10p complex binds to the transmembrane domains of the ADP/ ATP carrier. EMBO J. 21, 942-953.

Curran, S. P., Leuenberger, D., Schmidt, E., and Koehler, C. M. (2002b). The role of the Tim8p-Tim13p complex in a conserved import pathway for mitochondrial polytopic inner membrane proteins. J. Cell Biol. 257, 13028-13033.

Daum, G., Bohni, P. C., and Schatz, G. (1982). Import of proteins into mitochondria. Cytochrome $b_{2}$ and cytochrome $c$ peroxidase are located in the intermembrane space of yeast mitochondria. J. Biol. Chem. 257, 13028-13033.

Davis, A. J., Sepuri, N. B., Holder, J., Johnson, A. E., and Jensen, R. E. (2000). Two intermembrane space TIM complexes interact with different domains of Tim23p during its import into mitochondria. J. Cell Biol. 150, 1271-1282.

Davis, A. J., Alder, N. N., Jensen, R. E., and Johnson, A. E. (2007). The Tim9/10p and Tim8/13p complexes bind to specific sites on Tim23p during mitochondrial protein import. Mol. Biol. Cell 18, 475-486.

Dekker, P.J.T., Martin, F., Maarse, A. C., Bömer, U., Müller, H., Guiard, B., Meijer, M., Rassow, J., and Pfanner, N. (1997). The Tim core complex defines the number of mitochondrial translocation contact sites and can hold arrested preproteins in the absence of matrix Hsp70-Tim44. EMBO J. 16, 5408-5419.

Dolezal, P., Likic, V., Tachezy, J., and Lithgow, T. (2006). Evolution of the molecular machines for protein import into mitochondria. Science 313, 314318

D'Silva, P. D., Schilke, B., Walter, W., Andrew, A., and Craig, E. A. (2003). $\mathrm{J}$ protein cochaperone of the mitochondrial inner membrane required for protein import into the mitochondrial matrix. Proc. Natl. Acad. Sci. USA 100, $13839-13844$.

Endo, T., Yamamoto, H., and Esaki, M. (2003). Functional cooperation and separation of translocators in protein import into mitochondria, the doublemembrane bounded organelles. J. Cell Sci. 116, 3259-3267.

Farrell, S. R., and Thorpe, C. (2005). Augmenter of liver regeneration: a flavin-dependent sulfhydryl oxidase with cytochrome $c$ reductase activity. Biochemistry 44, 1532-1541.

Frazier, A. E., Chacinska, A., Truscott, K. N., Guiard, B., Pfanner, N., and Rehling, P. (2003). Mitochondria use different mechanisms for transport of multispanning membrane proteins through the intermembrane space. Mol. Cell Biol. 23, 7818-7828.

Gabriel, K., Milenkovic, D., Chacinska, A., Müller, J., Guiard, B., Pfanner, N. and Meisinger, C. (2007). Novel mitochondrial intermembrane space proteins as substrates of the MIA import pathway. J. Mol. Biol. 365, 612-620.

Herrmann, J. M., and Köhl, R. (2007). Catch me if you can! Oxidative protein trapping in the intermembrane space of mitochondria. J. Cell Biol. 176, 559563.

Hofhaus, G., Lee, J. E., Tews, I., Rosenberg, B., and Lisowsky, T. (2003). The N-terminal cysteine pair of yeast sulfhydryl oxidase Erv1p is essential for in vivo activity and interacts with the primary redox centre. Eur. J. Biochem. 270, $1528-1535$.

Hoogenraad, N. J., Ward, L. A., and Ryan, M. T. (2002). Import and assembly of proteins into mitochondria of mammalian cells. Biochim. Biophys. Acta $1592,97-105$.

Hoppins, S. C., and Nargang, F. E. (2004). The Tim8-Tim13 complex of Neurospora crassa functions in the assembly of proteins into both mitochondrial membranes. J. Biol. Chem. 279, 12396-12405.

Jensen, R. E., and Johnson, A. E. (2001). Opening the door to mitochondrial protein import. Nat. Struct. Biol. 8, 1008-1010.

Kadokura, H., Katzen, F., and Beckwith, J. (2003). Protein disulfide bond formation in prokaryotes. Annu. Rev. Biochem. 72, 111-135.

Koehler, C. M. (2004). The small Tim proteins and the twin $\mathrm{Cx}_{3} \mathrm{C}$ motif. Trends Biochem. Sci. 29, 1-4.

Koehler, C. M., Jarosch, E., Tokatlidis, K., Schmid, K., Schweyen, R. J., and Schatz, G. (1998). Import of mitochondrial carriers mediated by essential proteins of the intermembrane space. Science 279, 369-373.

Koehler, C. M., Leuenberger, D., Merchant, S., Renold, A., Junne, T., and Schatz, G. (1999). Human deafness dystonia syndrome is a mitochondrial disease. Proc. Natl. Acad. Sci. USA 96, 2141-2146.

Lee, J., Hofhaus, G., and Lisowsky, T. (2000). Erv1p from Saccharomyces cerevisiae is a FAD-linked sulfhydryl oxidase. FEBS Lett. 477, 62-66.

Lisowsky, T. (1994). ERV1 is involved in the cell-division cycle and the maintenance of mitochondrial genomes in Saccharomyces cerevisiae. Curr. Genet. 26, 15-20.

Lu, H., Allen, S., Wardleworth, L., Savory, P., and Tokatlidis, K. (2004a). Functional TIM10 chaperone assembly is redox-regulated in vivo. J. Biol. Chem. 279, 18952-18958.

Lu, H., Golovanov, A. P., Alcock, F., Grossmann, J. G., Allen, S., Lian, L. Y., and Tokatlidis, K. (2004b). The structural basis of the TIM10 chaperone assembly. J. Biol. Chem. 279, 18959-18966.

Lu, H., and Woodburn, J. (2005). Zinc binding stabilizes mitochondrial Tim10 in a reduced and import-competent state kinetically. J. Mol. Biol. 353, 897-910.

Luciano, P., Vial, S., Vergnolle, M. A., Dyall, S. D., Robinson, D. R., and Tokatlidis, K. (2001). Functional reconstitution of the import of the yeast ADP/ATP carrier mediated by the TIM10 complex. EMBO J. 20, 4099-4106.

Lutz, T., Neupert, W., and Herrmann, J. M. (2003). Import of small Tim proteins into the mitochondrial intermembrane space. EMBO J. 22, 44004408 .

Matouschek, A., and Glick, B. S. (2001). Barreling through the outer membrane. Nat. Struct. Biol. 8, 284-286.

Meisinger, C. et al. (2004). The mitochondrial morphology protein Mdm10 functions in assembly of the preprotein translocase of the outer membrane. Dev. Cell 7, 61-71. 
Meisinger, C., Pfanner, N., and Truscott, K. N. (2006). Isolation of yeast mitochondria. Methods Mol. Biol. 313, 33-39.

Meisinger, C. et al. (2007). The morphology proteins Mdm12/Mmm1 function in the major $\beta$-barrel assembly pathway of mitochondria. EMBO J. 26, 22292239.

Mesecke, N., Terziyska, N., Kozany, C., Baumann, F., Neupert, W., Hell, K., and Herrmann, J. M. (2005). A disulfide relay system in the intermembrane space of mitochondria that mediates protein import. Cell 121, 1059-1069.

Milenkovic, D., Gabriel, K., Guiard, B., Schulze-Specking, A., Pfanner, N., and Chacinska, A. (2007a). Biogenesis of the essential Tim9-Tim10 chaperone complex of mitochondria: site-specific recognition of cysteine residues by the intermembrane space receptor Mia40. J. Biol. Chem. 282, 22472-22480.

Milenkovic, D., Müller, J., Stojanovski, D., Pfanner, N., and Chacinska, A. (2007b). Diverse mechanisms and machineries for import of mitochondrial precursors. Biol. Chem. 388, 891-897.

Model, K., Meisinger, C., Prinz, T., Wiedemann, N., Truscott, K. N., Pfanner, N., and Ryan, M. T. (2001). Multi-step assembly pathway of the protein import channel of the mitochondrial outer membrane. Nat. Struct. Biol. 8 361-370.

Naoé, M., Ohwa, Y., Ishikawa, D., Ohshima, C., Nishikawa, S., Yamamoto, H., and Endo, T. (2004). Identification of Tim40 that mediates protein sorting to the mitochondrial intermembrane space. J. Biol. Chem. 279, 47815-47821.

Neupert, W., and Herrmann, J. M. (2007). Translocation of proteins into mitochondria. Annu. Rev. Biochem. 76, 723-749.

Oka, T., and Mihara, K. A. (2005). A railroad switch in mitochondrial protein import. Mol. Cell 18, 145-146.

Paschen, S. A., Rothbauer, U., Kaldi, K., Bauer, M. F., Neupert, W., and Brunner, M. (2000). The role of the TIM8 -13 complex in the import of Tim23 into mitochondria. EMBO J. 19, 6392-6400.

Prokisch, H. et al. (2004). Integrative analysis of the mitochondrial proteome in yeast. PLoS Biol. 2, e160.

Rapaport, D., Taylor, R. D., Käser, M., Langer, T., and Neupert, W. (2001). Structural requirements of Tom 40 for assembly into preexisting TOM complexes of mitochondria. Mol. Biol. Cell 12, 1189-1198.

Rehling, P., Brandner, K., and Pfanner, N. (2004). Mitochondrial import and the twin-pore translocase. Nat. Rev. Mol. Cell Biol. 5, 519-530.

Rissler, M., Wiedemann, N., Pfannschmidt, S., Gabriel, K., Guiard, B., Pfanner, N., and Chacinska, A. (2005). The essential mitochondrial protein Erv1 cooperates with Mia40 in biogenesis of intermembrane space proteins. J. Mol. Biol. 353, 485-492.

Schägger, H., and von Jagow, G. (1987). Tricine-sodium dodecyl sulfatepolyacrylamide gel electrophoresis for the separation of proteins in the range from 1 to $100 \mathrm{kDa}$. Anal. Biochem. 166, 368-379.
Schägger, H., and von Jagow, G. (1991). Blue native electrophoresis for isolation of membrane protein complexes in enzymatically active form. Anal. Biochem. 199, 223-231.

Sevier, C. S., and Kaiser, C. A. (2006). Conservation and diversity of the cellular disulfide bond formation pathways. Antioxid. Redox Signal 8, 797811

Sickmann, A. et al. (2003). The proteome of Saccharomyces cerevisiae mitochondria. Proc. Natl. Acad. Sci. USA 100, 13207-13212.

Sideris, D. P., and Tokatlidis, K. (2007). Oxidative folding of small Tims is mediated by site-specific docking onto Mia40 in the mitochondrial intermembrane space. Mol. Microbiol. 65, 1360-1373.

Sikorski, R. S., and Hieter, P. A. (1989). System of shuttle vectors and yeast host strains designed for efficient manipulation of DNA in Saccharomyces cerevisiae. Genetics 122, 19-27.

Sirrenberg, C., Endres, M., Fölsch, H., Stuart, R. A., Neupert, W., and Brunner, M. (1998). Carrier protein import into mitochondria mediated by the intermembrane proteins Tim10/Mrs11 and Tim12/Mrs5. Nature 391, 912-915.

Terziyska, N., Lutz, T., Kozany, C., Mokranjac, D., Mesecke, N., Neupert, W., Herrmann, J. M., and Hell, K. (2005). Mia40, a novel factor for protein import into the intermembrane space of mitochondria is able to bind metal ions. FEBS Lett. 579, 179-184.

Terziyska, N., Grumbt, B., Bien, M., Neupert, W., Herrmann, J. M., and Hell, K. (2007). The sulfhydryl oxidase Erv1 is a substrate of the Mia40-dependent protein translocation pathway. FEBS Lett. 581, 1098-1102.

Tokatlidis, K. (2005). A disulfide relay system in mitochondria. Cell 121, 965-967.

Truscott, K. N., Wiedemann, N., Rehling, P., Müller, H., Meisinger, C. Pfanner, N., and Guiard, B. (2002). Mitochondrial import of the ADP/ATP carrier: the essential TIM complex of the intermembrane space is required for precursor release from the TOM complex. Mol. Cell Biol. 22, 7780-7789.

Tu, B. P., and Weissman, J. S. (2004). Oxidative protein folding in eukaryotes: mechanisms and consequences. J. Cell Biol. 164, 341-346.

Vial, S., Lu, H., Allen, S., Savory, P., Thornton, D., Sheehan, J., and Tokatlidis, K. (2002). Assembly of Tim9 and Tim10 into a functional chaperone. J. Biol Chem. 277, 36100-36108.

Webb, C. T., Gorman, M. A., Lazarou, M., Ryan, M. T., and Gulbis, J. M (2006). Crystal structure of the mitochondrial chaperone TIM9-10 reveals a six-bladed $\alpha$-propeller. Mol. Cell 21, 123-133.

Wiedemann, N., Truscott, K. N., Pfannschmidt, S., Guiard, B., Meisinger, C., and Pfanner, N. (2004). Biogenesis of the protein import channel Tom40 of the mitochondrial outer membrane: intermembrane space components are in volved in an early stage of the assembly pathway. J. Biol. Chem. 279, 188-194. Wiedemann, N., Pfanner, N., and Chacinska, A. (2006). Chaperoning through the mitochondrial intermembrane space. Mol. Cell 21, 145-148.

Wilkinson, B., and Gilbert, H. F. (2004). Protein disulfide isomerase. Biochim. Biophys. Acta 1699, 35-44. 